\title{
Article \\ Application of Multi Network Alignment Algorithms for Connectomes Study
}

\author{
Marianna Milano ${ }^{1, \ddagger}$, Pietro Hiram Guzzi ${ }^{1, \ddagger *}$ and Mario Cannataro ${ }^{1}$ \\ 1 Department of Surgical and Medical Sciences, University of Catanzaro; m.milano,hguzzi,cannataro@unicz.it \\ * Correspondence: hguzzi@unicz.it \\ $\ddagger$ These authors contributed equally to this work.
}

\begin{abstract}
A growing area in neurosciences is focused on the modeling and analysis the complex system of connections in neural systems, i.e. the connectome. Here we focus on the representation of connectomes by using graph theory formalisms. The human brain connectomes are usually derived from neuroimages; the analyzed brains are co-registered in the image domain and brought to a common anatomical space. An atlas is then applied in order to define anatomically meaningful regions that will serve as the nodes of the network - this process is referred to as parcellation. Recently, it has been proposed to perform atlas-free random brain parcellation into nodes and align brains in the network space instead of the anatomical image space to define network nodes of individual brain networks. In the network domain, the question of comparison of the structure of networks arises. Such question is tackled by modeling the comparison of brain network as a network alignment (NA) problem. In this paper, we first defined the NA problem formally, then we applied three existing state of the art of multiple alignment algorithms (MNA) on diffusion MRI-derived brain networks and we compared the performances. The results confirm that MNA algorithms may be applied in cases of atlas-free parcellation for a fully network-driven comparison of connectomes.
\end{abstract}

Keywords: graph alignment; brain network; human connectome

\section{Introduction}

The human brain is a complex organ organized into a dense system of connections, also known as connectome. Recent studies have shown that this system of connections is responsible for the brain activity, and an alteration of connections (decreased or increased connectivity) can led to the insurgence of neurological diseases [1,2]. For this reasons, many researches in neuroscience have been focused on mapping and analysis of the human brain connectome [3]. Connectome may be analyzed using different zoom, e.g. by focusing on single components, i.e. neurons and axons, or grouping them into regions. Usually the analysis of single components is defined to as anatomic connectivity, while the analysis of regions is called functional connectivity because regions are in general perfoming different functions. Tipically, the human brain connectome can be mapped using neuroimaging techniques, such as Magnetic Resonance Imaging (MRI), Electroencephalography (EEG), and Electromyography (MEG) enabling to take a picture of the brain connections of patients [4]. Among the others, the main source for deriving information about connectomes is Magnetic Resonance Imaging (MRI) [5] able to achieve both informations about anatomic connectivity and functional connectivity.

Once obtained, the connectome data need to be characterized through sophisticated analytic strategies. A useful strategy is based on graph theory [6], that ensures the modeling of such data into a network model. Different studies have used the network models to extract clinically relevant information $[7,8]$ due to the capability to summarize the characteristics of a complex network with few measures and to understand the organization of both entire networks and individual network elements [6]. 
Graph theoretical approaches model the human brain as set of nodes linked by edges. The nodes typically represent region of interest (ROI) and the edges represent functional or anatomical connections. A typical MRI experiment produces a series of images, either from intra-subject or inter-subject, then the MRI images are modeled as networks. A further step consists of the coregistration among the network and a brain atlas in order to obtain anatomically meaningful regions [9]. Recently, Tymofiyeva et al. [10] proposed an alternative method based on the application of atlas-free parcellation and on the construction of individual connectomes only in the network space. In the network domain, an appropriate analytic strategy consists of the comparison of studied networks by recurring to network alignment (NA) approaches. The techniques for the alignment of biological networks fall into sub-categories: local alignment, to find small conserved motifs across networks, and global alignment, which attempts to find a best mapping between all nodes of the two networks or pairwise alignment to align two networks and multiple alignment that align multiple networks. Different studies have widely usad the NA approaches for the analysis of biological networks. In previous works $[11,12]$ we explored the possibility to apply the NA methods for the analysis of to MRI connectomics. At first we tested different global alignment algorithms to build the alignments among the diffusion MRI-derived brain networks. Then, we analyzed the alignment results in term of topological quality measures and according to these analyses, we identified the best alignment algorithm to align the diffusion MRI-derived brain networks. However, recent studies have demonstrated in an independent way that the multiple alignment algorithms are able to exact deeper information than pairwise alignment algorithm when these one are applied to molecular biology analysis [13].

According to these studies, we choose to apply multiple alignment algorithms on MRI connectomics. Here, we selected three existing state of the art multiple alignment algorithms to build the alignment of diffusion MRI-derived brain networks. The algorithms tested here are MultiMAGNA++ [13], GEDEVO-M [14] and IsoRankN [15]. The algorithms are applied to build the multiple alignment among the diffusion MRI-derived brain networks. After the alignments were built, we compared the performance of these algorithms.

\section{Methodology for Brain Network Analysis}

In this section, we present the workflow of analysis that can be preformed on the brain network starting from the building of connectome from MRI images. The Figure 1 shows a workflow of Methodology for the Brain Network Analysis from the building of representative network from experimental data to the comparison of brain network applying multiple alignment algorithm.

\subsection{Building a Brain Network}

The building of brain network starts with a set of anatomical or physiological observations [16], then the structural and functional connectivity data are processed into network model exploiting graph theory.

However, the application of graph theory to the study of connectomes presents some challenges related to the description of nodes and edges [17]. For example, an ideal definition of nodes should group a set of neurons according to maximal functional homogeneity within nodes and the maximal functional heterogeneity among different nodes. According to this, there is no clear evidence for the optimal definition of both nodes and edges. A common approach to define the nodes of a brain network consists of the subdivision of the brain into homogeneous, non-overlapping and large-scale, regions respect to information provided, generally, by techniques based on magnetic resonance imaging (MRI) [18], also known as "parcellation process". Especially, MRI has allowed to obtain information about anatomical connectivity, functional connectivity, or task-related activation.

Currently, there exist three different approaches applied to parcellation of connectome:

1. Parcellation of the brain by using predefined anatomical templates that consists of the registration of the structural images from MRI to anatomical atlas based on the Brodmann 


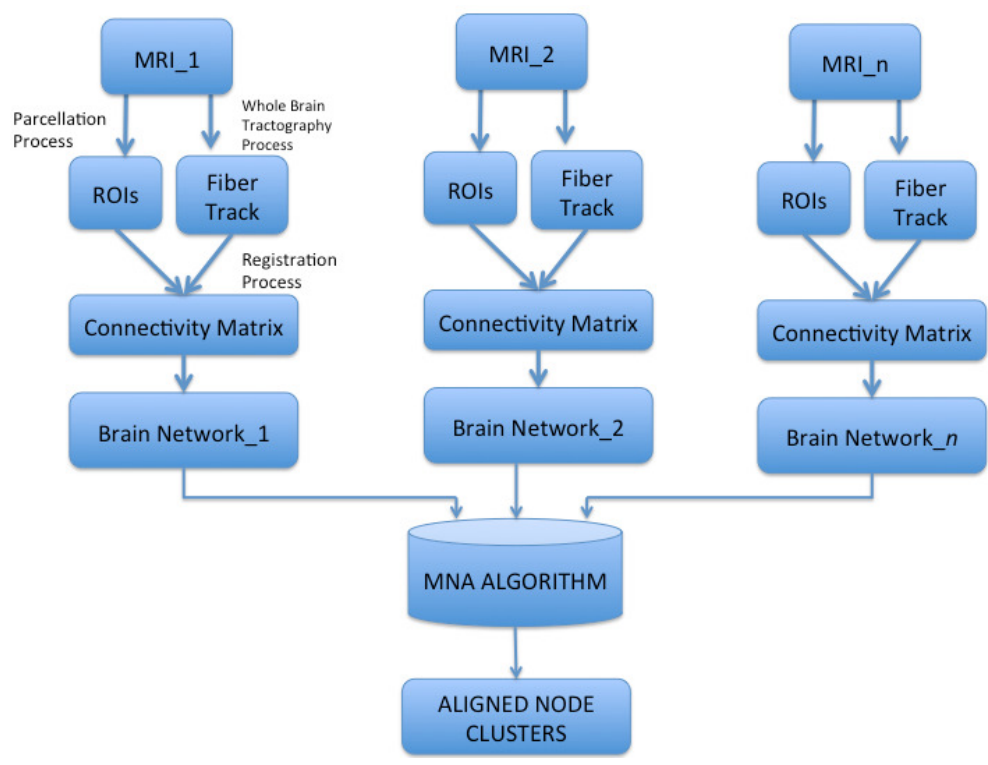

Figure 1. Building a representative network from experimental data: example of a workflow. Diffusion or functional MRI images are acquired for a subject according to the study to be conducted. The MRIs are used to perform whole-brain parcellation by selecting a suitable method. Starting from the parcelled whole brain the computation of connections is performed the connectivity matrix is constructed. Then, the resulting brain network is obtained. This process is preformed for each studied subject. A MNA algorithms takes as input the brain networks and produces aligned node clusters between more than two networks.

areas [19]. This approach enables to subdivide the whole brain into labeled regions according to the different labels regions of the templates;

2. Parcellation of the brain by using randomly generated templates [20] that ensures to divide the whole brain into parcels (brain region) of roughly equal size;

3. Connectivity-based parcellations that aim to delineate brain regions according to the similarities in structural or functional connectivity patterns.

Due to the different approach, the choice of a parcellation scheme is fundamental for subsequent analysis on brain network. In fact, each parcellation method presents some pitfalls.

For example, the parcellation of the brain by using predefined anatomical template raises the question of the accuracy of mapping. Since atlas based on the Brodmann areas are originally defined using cytoarchitectural differences between brain regions, in the registration step a mismatch among the cortical surface analyzed and the borders of the Brodmann areas may occurs [10].Thus, this approach is limited by inter-subject variability and can be especially problematic in the context of brain maturation. In this paper, we focus on the random, atlas-free definition of nodes in individual subjects (see [12] for a deep description), which can allow for a fully network-driven way of looking at the brain and comparing brains of different subjects and, potentially, species [10].

The definition of the edges is also currently an open challenge related to a) the type of connectivity measured, and b) the method used to quantify it. As mentioned above, brain connectivity can refer to different aspects of brain organization including (i) anatomical connectivity consisting of axonal fibers connecting cortical and subcortical regions inferred from diffusion imaging, and (ii) functional connectivity defined as the observed statistical correlations of the BOLD signal between brain regions.

Once the nodes and the edges are defined, the pattern of connections between brain regions (nodes) can be stored into the Connectivity Matrix [21]. The Connectivity Matrix is symmetric matrix where rows and columns represent different brain regions, and the entries correspond to connection 
(edge) between the regions. This representation lends itself to be mapped to a graphical model which ensures to quantify different topological aspects of the connectome.

\subsection{Comparison of Brain Network: Network Alignment}

A crucial point in the connectome analysis regards the comparison of the brain networks. Thus, the detection of an correct node mapping between atlas-free networks may uncover significant aspcets on the comparison of brains or structure of groups of subjects, such as healthy versus diseased subjects. Many different network alignment methods have been proposed in biological fields [22].

Formally, a graph $G$ is defined as $G=\{V, E\}$, where $V$ is a finite set of nodes and $E$ is a finite set of edges. Let $G_{1}=\left\{V_{1}, E_{1}\right\}$ and $G_{2}=\left\{V_{2}, E_{2}\right\}$ be two graphs, where $V_{1,2}$ are sets of nodes and $E_{1,2}$ are sets of edges, a graph alignment is the mapping between the nodes of the input networks that maximizes the similarity between mapped entities. From a theoretical point of view, the graph alignment problem consists of finding an alignment function (or a mapping) $f: V_{1} \rightarrow V_{2}$ that maximizes a cost function $Q$. The similarity between the graphs is defined by a cost function, $Q\left(G_{1}, G_{2}, f\right)$, also known as the quality of the alignment. Let $f$ be an alignment between two graphs $G_{1}$ and $G_{2}$, given a node $u$ from $G_{1}, f(u)$ is the set of nodes from $G_{2}$ that are aligned under $f$ to $u$. Q expresses the similarity among two input graphs with respect to a specific alignment $f$ and the formulation of $Q$ strongly influences the mapping strategy.

There exist different formulations of $\mathrm{Q}$ that fall into following the classes:

Topological Similarity: Graphs are aligned by considering only edge topology, so that the perfect alignment is reached when input graphs are isomorphic.

Node Similarity: Such function considers the similarity among mapped nodes. Nodes of the aligned graphs can be more or less similar to each other. Thus the alignment should align each node of one graph to the most similar node of the other one given a node similarity functions, $s\left(v_{1}, v_{2}\right) \rightarrow R$, $v_{1} \in V_{1}, v_{2} \in V_{2}$.

Hybrid approaches: Some recent formulations of $Q$ take into account of both of the approaches by linear combination.

The network alignment problem can be formulated according to: i) the kind of input, pairwise or multiple alignment and ii) the scope of node mapping required, local or global alignment. In general, the network alignment can be classified as local alignment or global alignment. The local alignment typically finds multiple and unrelated regions of isomorphism among the input networks, each region implying a mapping independently of the others. Therefore, the computed correspondences may involve overlapping subgraphs. The output of local network alignment is a set of pairs of possibly overlapping subgraphs of the input networks. The literature contains many algorithms that address local graph alignment problem. For example, AlignNemo [23] and AlignMCL [24] algorithms. The global alignment aims to find a mapping that should cover all of the nodes of the input networks. Global alignment returns a unique overall alignment between the input networks, such that a one-to-one correspondence is found between of a network with one node of the other network. Most popular existing methods of global alignment are MAGNA [25], NETAL [26], GHOST [27], WAVE [28]. For a complete review on global and local network alignment algorithms and their advantages or disadvantages see [29].

Also, the network alignment methods can be pairwise or multiple alignment.

The pairwise network alignment (PNA) aligns two networks at a time and produces aligned node pairs between two networks. The multiple network alignment (MNA) aligns three or more networks to each other at once and produces aligned node clusters. PNA and MNA can be local or global, with one-to-one or many-to-many node mappings. The difference between one-to-one and many-to-many mapping in the pairwise alignment refers the previous discussion on global and local alignment. The PNA can search the similar small subnetworks exploiting many-to-many mapping between nodes of the compared network or can look for the best overlap of the whole compared networks exploiting one-to-one node mapping. MNAs are one-to-one MNA methods when produce an aligned cluster 
containing at most one node per network, whereas MNAs are many-to-many MNA methods when an aligned cluster contains more than one node from a single network.

In literature, both PNA and MNA are applied to built the alignment protein interaction networks (PINs) [30]. Since, MNA can capture functional knowledge that is common to multiple species, it was detected that MNA leads to deeper biological information than PNA. However, MNA is computationally much harder than PNA because the complexity of the network alignment problem increases exponentially with the number of analyzed networks.

There exist different proposed multiple network alignment algorithms in literature such as MultiMAGNA++ [13], GEDEVO-M [14] and IsoRankN [15].

In this work, three multiple alignment algorithms were chosen to built the multiple alignment of brain networks. We give hereafter a short conceptual description.

A popular existing method of multiple alignment is MultiMAGNA++ [13]. MultiMAGNA++ is a a global one-to-one MNA aligner that simulates a population of alignments that evolves over time by applying a genetic algorithm and a function for the crossover of two alignments into a superior alignment. Since the genetic algorithm simulates the evolutionary process guided by the survival of the fittest principle, only alignments, i.e. those that conserve the most edges, survive. Thus, MultiMAGNA++ proceeds to the next generation, until the alignment accuracy cannot be optimized further.

The second multiple aligner is GEDEVO-M [14] a global one-to-one MNA aligner. GEDEVO-M is an extension of GEDEVO [31] tool for efficient global graph alignment. Underlying the GEDEVO-M method is the Graph Edit Distance model (GED), where a graph is transferred into another one with a minimal number of edge insertions and deletions. Thus, GEDEVO-M uses the GED as optimization model for finding the best alignments and then minimizes the sum of GEDs between every pair of input networks.

The last multiple aligner is IsoRankN [15], a global many-to-many MNA alignment tool based a spectral clustering method to find dense and clique modules when the global alignment of multiple networks is computed.

\section{Results}

\subsection{Dataset}

The dataset consisted of 24 diffusion MRI-derived structural networks of human brain: 12 networks with a number of nodes equal to 95 and the 12 networks with a number of nodes equal to 1000. The brain networks are related to three different stages of development by including newborns (NE), six-month-old infants (6M), and adults (AD). See Materials and Methods Section for a complete description.

\subsection{Building of brain network multiple alignment}

We built the multiple alignment of all networks with 95 and 1000 nodes (for convenience we call the two dataset networks ${ }_{95}$ and networks ${ }_{1000}$ ) related to same growth stages (NE, 6M, AD) by applying MultiMAGNA++ [13], GEDEVO-M [14] and IsoRankN [15].

We ran all MNA methods on the same Linux machine with Intel Core i5 and 4GB of RAM. We selected the following MultiMAGNA++ parameters: $C I Q$ as measure of Edge Conservation, the $\alpha$ parameter equal to 0 , in order to consider only topology, whereas the population size, number of generation, fraction of elite members were set to default values. We tested different parameters and obtained best results with the default parameters for GEDEVO-M: pop parameter that controls the number of new individuals per iteration set equal to 1000 and maxsame that controls the stop after $\mathrm{N}$ iterations without any score improvement were equal to 3000. To build the multiple alignment with IsoRankN we set: the max number of iterations $K$ equals to 30 , the threshold thresh equals to $1 e-4$, maxveclen equals to 1000000 and the $\alpha$ parameter equal to 1 in order to consider only network data. 
The Table 1 reports the execution time of MultiMAGNA++, GEDEVO-M and IsoRankN to build the multiple alignment on the networks with 95 nodes and on the networks with 1000 nodes.

Table 1. Execution Time to build the multiple alignment with MultiMAGNA++, GEDEVO-M and IsoRankN for the networks with 95 nodes and the networks with 1000 nodes

\begin{tabular}{lllll}
\hline & $\begin{array}{l}\text { Execution Time for } \\
\text { network with 95 nodes }\end{array}$ & $\begin{array}{l}\text { Execution Time for } \\
\text { network with 95 nodes }\end{array}$ & Processor & Memory \\
\hline MultiMAGNA++ & 5 seconds & 7 seconds & Intel Core i5 & 4 GB \\
\hline GEDEVO-M & 8 seconds & 11 seconds & Intel Core i5 & 4 GB \\
\hline IsoRankN & 6 seconds & 10 seconds & Intel Core i5 & 4 GB \\
\hline
\end{tabular}

\subsection{Topological alignment quality evaluation}

Here, we aim to evaluate the quality of the multiple alignments built with MultiMAGNA++, GEDEVO-M and IsoRankN algorithms. The topological quality is related to two alignment algorithm capability as the reconstruction of the true node mapping and the conservation of as much as possible edges. Typically, the Node Correctness (NC) is the measure widely used to evaluate how an alignment reconstructs the true node mapping correctly. Instead, different measures are used to evaluate how well the edges are conserved on an alignment, such as EC, ICS or $S^{3}$ (see the previous Section). In general, the Edge Correctness is defined as the number of edges conserved under an alignment $f$ with respect to the total number of edges of input networks. Thus, once the multiple alignments were built, we performed an evaluation of alignment quality by comparing the Edge Correctness (EC) [25] related to the alignments built with MultiMAGNA++, GEDEVO-M and IsoRankN.

The Table 2 and Table 3 report the global Edge Correctness computed on the multiple alignment of all networks with 95 nodes and with 1000 nodes related to same growth stages NE, 6M, AD by applying MultiMAGNA++, GEDEVO-M and IsoRankN algorithms.

Table 2. Comparison the Edge Correctness of the multiple alignments built with MultiMAGNA++, GEDEVO-M and IsoRankN.

\begin{tabular}{|l|l|l|l|}
\hline Edge Correctness & NE & $\mathbf{6 M}$ & AD \\
\hline MultiMAGNA++ & $\mathbf{0 . 5}$ & $\mathbf{0 . 5 5}$ & $\mathbf{0 . 4 9}$ \\
GEDEVO-M & 0.441 & 0.441 & 0.48 \\
IsoRankN & 0.477 & 0.477 & 0.485 \\
\hline
\end{tabular}

Table 3. Comparison the Edge Correctness of the multiple alignments built with MultiMAGNA++, GEDEVO-M and IsoRankN.

\begin{tabular}{|l|l|l|l|}
\hline Edge Correctness & NE & $\mathbf{6 M}$ & AD \\
\hline MultiMAGNA++ & $\mathbf{0 . 1 4}$ & $\mathbf{0 . 1 6}$ & $\mathbf{0 . 1 9}$ \\
GEDEVO-M & 0.089 & 0.091 & 0.099 \\
IsoRankN & 0.095 & 0.099 & 0.1 \\
\hline
\end{tabular}

Figure 2 shows an overview of edge conservation comparison on networks $s_{95}$ whereas Figure 3 shows an overview of edge conservation comparison on networks 1000 . We note that the best results in terms of edge conservation were obtained when applying MultiMAGNA++ as global aligner both on networks $s_{95}$ and networks $s_{1000}$. In fact, the mean edge correctness values are higher on the alignments built with MultiMAGNA++ than mean edge correctness scores on alignments obtained with GEDEVO-M and IsoRankN. The reason is related to the strategy of MultiMAGNA++ to construct the multiple alignment. In fact, MultiMAGNA++ is the unique multiple aligner that directly optimizes edge conservation in addition to node conservation by using a genetic algorithm, whereas the MNA algorithms optimize node conservation only. In this way, the quality of alignment built with 
MultiMAGNA++ results improved. This entails an inferior behavior of GEDEVO-M and IsoRankN compared to the MultiMAGNA++.

We also note that values of EC for networks ${ }_{95}$ are higher than EC for networks 1000 .

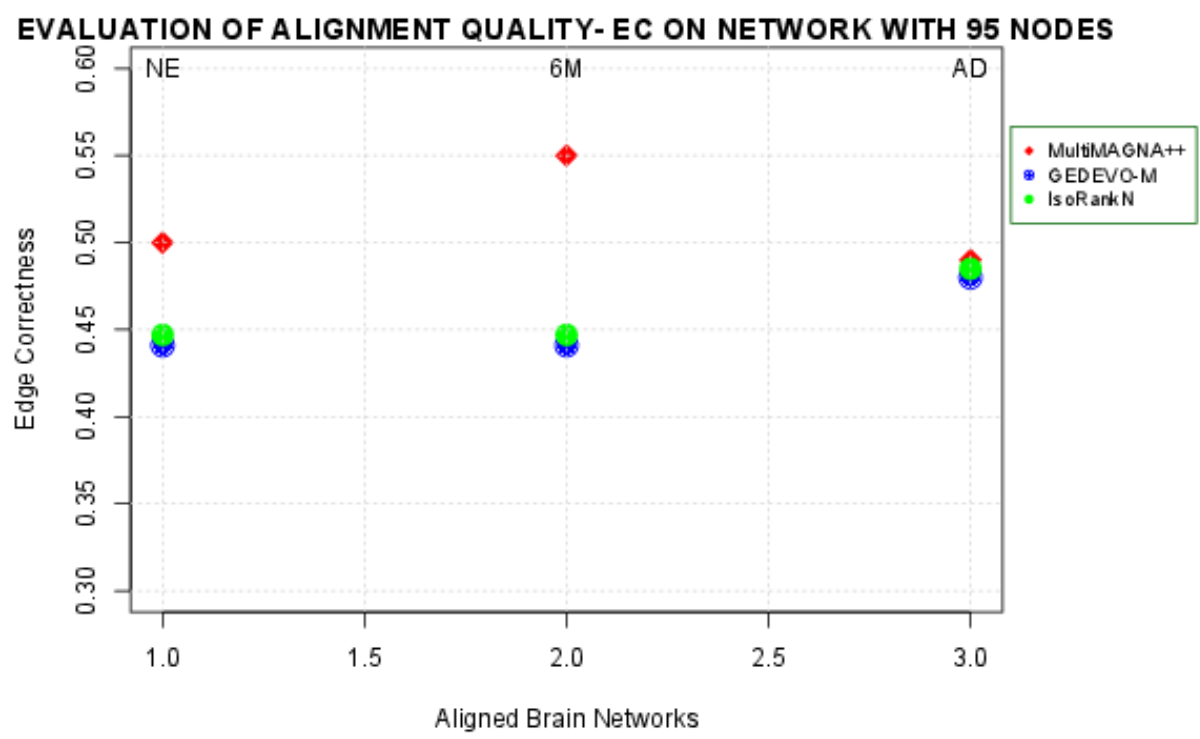

Figure 2. The topological evaluation of alignments built with MultiMAGNA++ (red marker), GEDEVO-M (blue marker), IsoRankN (green marker). The Figure shows the mean Edge Correctness scores of alignments built among the networks with 95 nodes by applying the selected three multiple aligners.

\section{Discussion}

The brain connectivity refers to different aspects of brain organization including i) anatomical connectivity consisting of axonal fibers across cortical regions and ii) functional connectivity defined as the observed statistical correlations of the BOLD signal between regions of interest. Understanding brain connectivity can shed light on the brain cognitive functioning that occurs via the connections and interaction between neurons. Brain connectivity can be modeled and quantified with a large number of techniques. A useful formalism to represent the brain connectivity derives from graph theory. The graph theoretical modeling of the human connectome has enabled important discoveries by comparing the brain networks of studied subjects. In this study we proposed to apply three multiple alignment algorithms MultiMAGNA++, GEDEVO-M and IsoRankN to align atlas-free human brain networks at three developmental stages. We decided to apply MNA algorithms to the study of brain networks because, in previous studies conducted on PINs, MNA were able to lead to deeper biological compared to PNA, by capturing conserved network regions between multiple networks. We analyzed the multiple alignment results in term of topological quality measures, by comparing the EC related to each alignment. According to these analyses, MultiMAGNA++ resulted in the best alignment. The reason is related to the strategy underlying MultiMAGNA++ to construct the multiple alignment by optimizing simultaneously the edge conservation and node conservation. Our ongoing study is focused on the implementation of an ad hoc algorithm for connectome alignment. Since there are many conditions in which the classical parcellation is not useful, we retain that this seminal work may open the way for the use of multiple network alignment in atlas-free parcellation. 


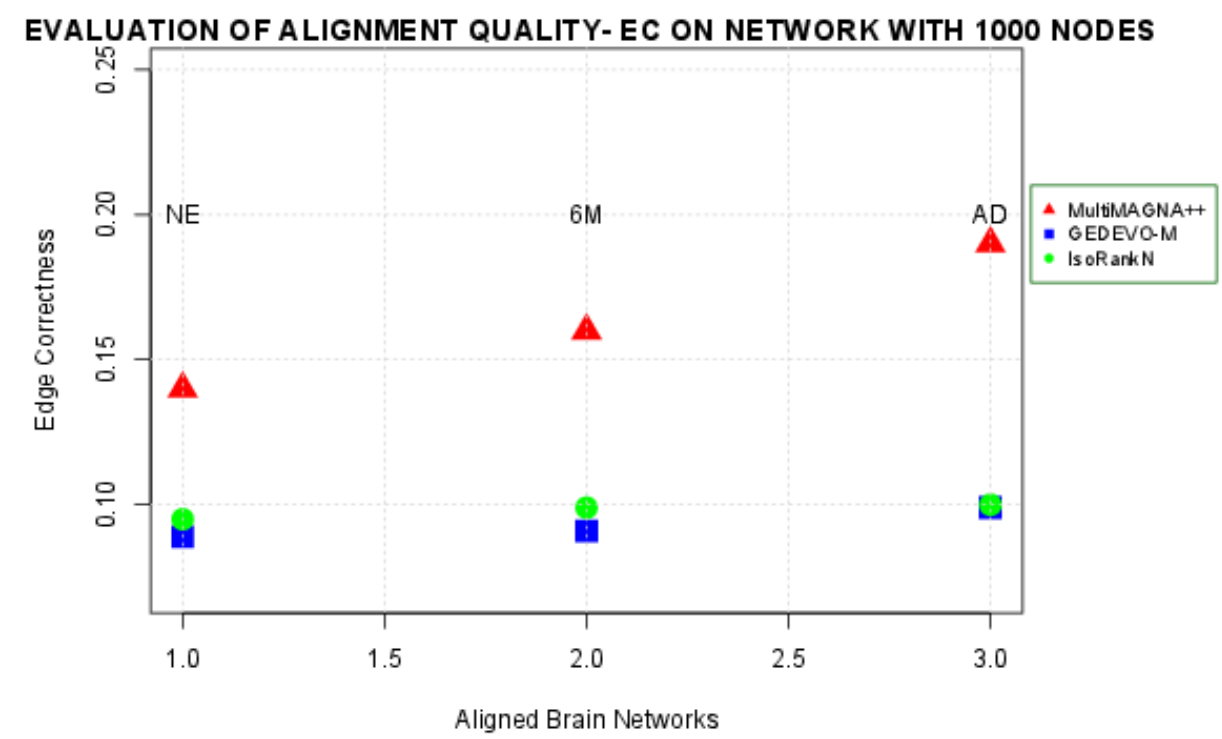

Figure 3. The topological evaluation of alignments built with MultiMAGNA++ (red marker), GEDEVO-M (blue marker), IsoRankN (green marker). The Figure shows the mean Edge Correctness scores of alignments built among the networks with 1000 nodes by applying the selected three multiple aligners.

\section{Materials and Methods}

\subsection{Dataset}

The dataset consisted of diffusion MRI-derived structural networks of human brain at different stages of development, starting with neonates [10]. Acquisition of the MRI data was compliant with the Health Insurance Portability and Accountability Act (HIPAA) and the study was approved by the Committee on Human Research (CHR) of the University of California, San Francisco. Three age groups were included: 4 neonates imaged in the first 4-5 days of life (NE), 4 six-month-old infants (6M), and 4 adults (age 24-31 years) (AD). The two pediatric groups had transient encephalopathy at birth, but none of the patients had clinical or imaging evidence of brain injury. The subjects were scanned on a 3T GE MR scanner using a spin echo (SE) echo planar imaging (EPI) diffusion tensor imaging DTI sequence with parameter described in [10]. Tensor calculation, tractography, cortical parcellation into $\mathrm{N}$ equal-area nodes (Figure ??), and construction of the connectivity matrices was performed as described previously [10]. All networks were binarized with a threshold of 1 streamline. Starting from the images we obtained two different datasets. The first dataset consist of 12 networks with number of nodes equal to 95 depending on parcellation step. For convenience we call this dataset networks95. Table 4 shows the networks parameters. About the second dataset, the 12 networks were constructed by setting the number of equal-area nodes for the cortical parcellation equal to 1000 . Since all cortical areas of the brain are connected, a fine parcellation should ensure the interconnectedness of the whole brain, leaving no nodes isolated. In [10] the authors demonstrated that the highest number of nodes at which this condition is fulfilled in equal to 95. For this reason, the networks of the second dataset showed the isolated nodes that were not computed in the construction of the connectivity matrices. For convenience we call this dataset networks 1000 even though the nodes number is different from 1000. Table 5 shows the network parameters. 
Table 4. Details of brain networks with 95 nodes used for experiments

\begin{tabular}{ccc}
\hline Network & Nodes & Edge \\
\hline NE01 & 95 & 341 \\
NE02 & 95 & 341 \\
NE03 & 95 & 334 \\
NE04 & 95 & 320 \\
6M01 & 95 & 353 \\
6M02 & 95 & 333 \\
6M03 & 95 & 333 \\
6M04 & 95 & 338 \\
AD1 & 95 & 449 \\
AD2 & 95 & 406 \\
AD3 & 95 & 438 \\
AD4 & 95 & 416 \\
\hline
\end{tabular}

Table 5. Details of brain networks with 1000 nodes used for experiments

\begin{tabular}{ccc}
\hline Network & Nodes & Edge \\
\hline NE01 & 889 & 2555 \\
NE02 & 904 & 2618 \\
NE03 & 900 & 2585 \\
NE04 & 899 & 2298 \\
6M01 & 902 & 2458 \\
6M02 & 849 & 2182 \\
6M03 & 805 & 1928 \\
6M04 & 851 & 2087 \\
AD1 & 902 & 3146 \\
AD2 & 869 & 2691 \\
AD3 & 878 & 3262 \\
AD4 & 853 & 2907 \\
\hline
\end{tabular}

\subsection{Alignment Algorithms}

In this section we describe in detail the multiple alignment algorithms selected to align the diffusion brain networks.

MultiMAGNA++ [13] is a global one-to-one MNA algorithm based on a genetic algorithm to build an improved alignment. By simulating the evolutionary process, guided by the survival of the fittest principle, the genetic algorithm directly optimizes both edge and node conservation while the alignment is constructed. In details, MultiMAGNA++ uses the genetic algorithm to simulates a population of alignments that evolves over time and then applies new function for the crossover of parent alignments into a superior child alignment that allows for aligning multiple networks.

The genetic algorithm requires an initial population of a given number of members. In MultiMAGNA++, the members of population are multiple alignments. A multiple network alignment (MNA) of $k$ networks, ordered in terms of the number of nodes from the smallest to the largest one, is represented by using $k-1$ permutations which are bijective mappings between pairs of networks adjacent. The permutations are set of disjoint node clusters that cover nodes in the $k$ networks. So MNA can be defined as multi permutation. The members of a population crossover with each other to produce new members. Only the fitted members are more likely to crossover. Thus, the child alignment resulting from a crossover function reflects each parent. In MultiMAGNA++, the crossover function is defined as the midpoint of the shortest path between two permutations. In this way, the child MNA shares the characteristics of each of the two parent MNAs. To avoid the size of the population to grow without bound, the size is kept constant across all generations, with only the fittest members surviving from one generation to the next. The fitness function is a combined measure of edge conservation $S_{E}$ and node conservation $S_{N}$ maximized as follow: 


$$
\alpha S_{E}+(1-\alpha) S_{N}
$$

where $\alpha$ controls the contribution of each node and edge conservation measures and takes the values between 0 and 1 .

The edge conservation measure used in MNA is Conserved Interaction Quality (CIQ). CIQ is a weighted sum of edge conservation between all pairs of aligned $a$ and $b$ clusters and is defined as:

$$
S_{E}=C I Q=\frac{\sum_{a, b}\left|E_{a, b} b\right| c s(a, b)}{\sum_{a, b}\left|E_{a}, b\right|}
$$

where, $\left|E_{a}, b\right|$ is the number of edges that connect the clusters, and $\operatorname{cs}(a, b)$ is edge conservation between two clusters. Let $r(a, b)$ be the number of networks that the edges which connect the clusters belong to and $s(a, b)$ be the number of networks that contain at least one node in both clusters, $\operatorname{cs}(a, b)$ is equal to 0 if $r(a, b) \leq 1$, also $\operatorname{cs}(a, b)$ is equal to $\frac{r(a, b)}{s(a, b)}$.

The node conservation measure for MNA refers to internal cluster quality, i.e, the nodes in each cluster should be highly similar to each other with respect to some node cost function.

$$
S_{N}=\frac{1}{n} \sum_{i=1}^{n} \frac{1}{\left(\begin{array}{c}
a_{i} \mid \\
2
\end{array}\right)} \sum_{(u, v) \in P\left(a_{i}\right)} s(u, v)
$$

where $s(u, v)$ is the similarity between nodes $u$ and $v$ with respect to some node cost function, $a_{i}$ is a aligned clusters with $i=1, \ldots, n,\left|a_{i}\right|$ is the size of $a_{i}$ and $P\left(a_{i}\right)$ is the set of all pairs of nodes in $a_{i}$.

The genetic algorithm produces newer generations until the alignment quality cannot be optimized further.

GEDEVO-M [14] is a global one-to-one MNA algorithm based on an evolutionary algorithm that uses the Graph Edit Distance (GED) as optimization model for finding the best alignments. The GED is defined as the minimum insertions and deletions of edges required to transfer a graph into another graph. GEDEVO-M applies the Graph Edit Distance to multiple graph models and considers the alignment building as Topological Multiple one-to-one Network Alignment (TMNA). TMNA problem aims to find a multiple mapping $F$ on a set of graphs $G$, such that the multiple Graph Edit Distance $m G E D_{F}$ is minimal over all possible multiple mapping on $G$. By minimizing the $m G E D_{F}$, the number of edges that are aligned in multiple networks simultaneously is maximized. The GEDEVO-M builds the alignment by generating an initial multiple mapping with random permutations. A one-to-one MNA of $G$ graphs consists of a set of disjoint clusters. Each cluster can be represented as a tuple. Initially, GEDEVO-M fixes a threshold, defined as the average over all tuple scores and then it randomly swaps the tuples that have scores higher than the threshold. The tuples with lower than the threshold are also given a certain chance to be swapped. After that, GEDEVO-M uses a crossover operator to construct a new multiple mapping from two or more parent individuals of the previous generation. At first, GEDEVO-M computes the tuple scores for every possible subset of G. Then, GEDEVO-M iterates over the corresponding tuple scores by starting with larger subsets of $G$ and assigns some of these tuples to a new multiple mapping until every subset is considered. Finally, GEDEVO-M evaluates the quality of the multiple mapping by using the score $S$. The score $S$ depend on the multiple Graph Edit Distance ( $m G E D)$ and Graphlet-degree signature distance (GSD) of multiple mapping that computes the difference in neighboring topologies of potentially matched nodes.

IsoRankN [15] is a global many-to-many MNA alignment tool based a spectral partitioning method to find dense and clique clusters on multiple-network alignment.

IsoRankN builds a multiple network alignment by local partitioning the graph of pairwise functional similarity scores. Initially, IsoRankN computes the functional similarity scores of every pair of nodes of $k$ networks. In this way, a functional similarity graph, where each edge is weighted by its functional similarity score, is obtained. Then, IsoRankN applies a star spread method on functional similarity graph to obtain a multiple alignment as highly similar cliques. In detail, IsoRankN computes, 


$\begin{array}{ll}\text { NA } & \text { Network Alignment } \\ \text { PNA } & \text { Pairwise Network Alignment } \\ \text { MNA } & \text { Multiple Network Alignment } \\ \text { MRI } & \text { Magnetic Resonance Imaging } \\ \text { ROIs } & \text { Region of Interest } \\ \text { DTI } & \text { Diffusion Tensor Imaging } \\ \text { PINs } & \text { Protein Interaction Networks } \\ \text { BOLD } & \text { Blood Oxygenation Level Dependent } \\ \text { NE } & \text { Newborns } \\ 6 \text { M } & \text { Six-Month-Old } \\ \text { AD } & \text { Adults } \\ \text { HIPAA } & \text { Health Insurance Portability and Accountability Act } \\ \text { CHR } & \text { Committee on Human Research } \\ S^{3} & \text { Symmetric Substructure Score } \\ \text { EC } & \text { Edge Correctness } \\ S_{E} & \text { Edge Conservation } \\ S_{N} & \text { Node Conservation } \\ \text { CIQ } & \text { Conserved Interaction Quality } \\ \text { GED } & \text { Graph Edit Distance } \\ \text { TMNA } & \text { Multiple one-to-one Network Alignment } \\ m G E D_{F} & \text { Multiple Graph Edit Distance } \\ & \end{array}$
represent the star of a node $S$. to a cluster. All authors read and approved the final manuscript.

Conflicts of Interest: The authors declare no conflict of interest.

\section{Abbreviations}

The following abbreviations are used in this manuscript:

NA Network Alignment

PNA Pairwise Network Alignment

MNA Multiple Network Alignment

MRI Magnetic Resonance Imaging

ROIs Region of Interest

DTI Diffusion Tensor Imaging

PINs Protein Interaction Networks

Blood Oxygenation Level Dependent

Newborns

AD Adults

HIPAA Health Insurance Portability and Accountability Act

CHR Committee on Human Research

$S^{3} \quad$ Symmetric Substructure Score

EC Edge Correctness

$S_{E} \quad$ Edge Conservation

Node Conservation

$m G E D_{F} \quad$ Multiple Graph Edit Distance

\section{References} PLoS Comput Biol 2005, 1, e42. neuropsychiatric disorders. Brain connectivity 2011, 1, 349-365. Neurosurgery 2012, 71, 1.

for each node, every neighbor connected with an edge whose weight is greater than a threshold; this

Then, IsoRankN orders the nodes according to the total weight of the star $S$. For each the star $S$, a subset with highly weighted neighborhood is found. This subset represents a functionally conserved interaction cluster. Finally, IsoRankN performed a merging stars process, by looking at the neighbors of the neighbors of a node and by merging the stars of two nodes if every member of star related to a node 1 has the node 2 as a neighbor and vice versa. The process is repeated until all nodes are assigned

Acknowledgments: PHG,MM and MC have been partially supported by the following research project funded by the Italian Ministry of Education and Research (MIUR): BA2Know-Business Analytics to Know (PON03PE_00001_1). The authors wish to thank Olga Tymofiyeva for her suggestions to this research activity.

Author Contributions: PHG and MM conceived the main idea of the algorithm and designed the tests. MC supervised the design of the algorithm. PHG and MM designed the functional requirements of the software tool.

1. Kiani, R.; Cueva, C.J.; Reppas, J.B.; Peixoto, D.; Ryu, S.I.; Newsome, W.T. Natural grouping of neural responses reveals spatially segregated clusters in prearcuate cortex. Neuron 2015, 85, 1359-1373.

2. Bargmann, C.I.; Marder, E. From the connectome to brain function. Nature methods 2013, 10, 483-490.

3. Sporns, O.; Tononi, G.; Kötter, R. The human connectome: a structural description of the human brain.

4. Xia, M.; He, Y. Magnetic resonance imaging and graph theoretical analysis of complex brain networks in

5. Toga, A.W.; Clark, K.A.; Thompson, P.M.; Shattuck, D.W.; Van Horn, J.D. Mapping the human connectome. 
6. Bullmore, E.; Sporns, O. Complex brain networks: graph theoretical analysis of structural and functional systems. Nature Reviews Neuroscience 2009, 10, 186-198.

7. Cannataro, M.; Guzzi, P.H.; Veltri, P. Protein-to-protein interactions: Technologies, databases, and algorithms. ACM Computing Surveys (CSUR) 2010, 43, 1.

8. Lesne, A. Complex Networks: from Graph Theory to Biology. Letters in Mathematical Physics 2006 78, 235-262.

9. Yap, P.T.; Wu, G.; Shen, D. Human Brain Connectomics: Networks, Techniques, and Applications [Life Sciences]. IEEE Signal Processing Magazine 2010, 27, 131-134.

10. Tymofiyeva, O.; Ziv, E.; Barkovich, A.J.; Hess, C.P.; Xu, D. Brain without anatomy: construction and comparison of fully network-driven structural MRI connectomes. PloS one 2014, 9, e96196.

11. Milano, M.; Tymofiyeva, O.; Xu, D.; Hess, C.; Cannataro, M.; Guzzi, P.H. Using Network Alignment for Analysis of Connectomes: Experiences from a Clinical Dataset. Proceedings of the 7th ACM International Conference on Bioinformatics, Computational Biology, and Health Informatics. ACM, 2016, pp. 649-656.

12. Milano, M.; Guzzi, P.H.; Tymofieva, O.; Xu, D.; Hess, C.; Veltri, P.; Cannataro, M. An extensive assessment of network alignment algorithms for comparison of brain connectomes. BMC bioinformatics 2017, 18, 235.

13. Vijayan, V.; Milenkovic, T. Multiple network alignment via multiMAGNA++. arXiv preprint arXiv:1604.01740 2016.

14. Ibragimov, R.; Malek, M.; Baumbach, J.; Guo, J. Multiple graph edit distance: simultaneous topological alignment of multiple protein-protein interaction networks with an evolutionary algorithm. Proceedings of the 2014 Annual Conference on Genetic and Evolutionary Computation. ACM, 2014, pp. 277-284.

15. Liao, C.S.; Lu, K.; Baym, M.; Singh, R.; Berger, B. IsoRankN: spectral methods for global alignment of multiple protein networks. Bioinformatics 2009, 25, i253-i258.

16. Sporns, O. Networks of the Brain; MIT press, 2010.

17. Fornito, A.; Zalesky, A.; Breakspear, M. Graph analysis of the human connectome: promise, progress, and pitfalls. Neuroimage 2013, 80, 426-444.

18. Thirion, B.; Varoquaux, G.; Dohmatob, E.; Poline, J.B. Which fMRI clustering gives good brain parcellations? Frontiers in neuroscience 2014, 8, 167.

19. Geyer, S.; Weiss, M.; Reimann, K.; Lohmann, G.; Turner, R. Microstructural parcellation of the human cerebral cortex-from Brodmann's post-mortem map to in vivo mapping with high-field magnetic resonance imaging. Frontiers in human neuroscience 2011, 5, 19.

20. Fornito, A.; Zalesky, A.; Bullmore, E.T. Network scaling effects in graph analytic studies of human resting-state FMRI data. Resting state brain activity: Implications for systems neuroscience 2010, p. 40.

21. Rubinov, M.; Sporns, O. Complex network measures of brain connectivity: uses and interpretations. Neuroimage 2010, 52, 1059-1069.

22. Meng, L.; Striegel, A.; Milenkovic, T. Local versus Global Biological Network Alignment. arXiv preprint arXiv:1509.08524 2015.

23. Ciriello, G.; Mina, M.; Guzzi, P.H.; Cannataro, M.; Guerra, C. AlignNemo: A Local Network Alignment Method to Integrate Homology and Topology. PloS one 2012, 7, e38107.

24. Mina, M.; Guzzi, P.H. AlignMCL: Comparative analysis of protein interaction networks through Markov clustering. Bioinformatics and Biomedicine Workshops (BIBMW), 2012 IEEE International Conference on. IEEE, 2012, pp. 174-181.

25. Saraph, V.; Milenković, T. MAGNA: maximizing accuracy in global network alignment. Bioinformatics 2014, 30, 2931-2940.

26. Neyshabur, B.; Khadem, A.; Hashemifar, S.; Arab, S.S. NETAL: a new graph-based method for global alignment of protein-protein interaction networks. Bioinformatics 2013, 29, 1654-1662.

27. Patro, R.; Kingsford, C. Global network alignment using multiscale spectral signatures. Bioinformatics 2012, 28, 3105-3114.

28. Sun, Y.; Crawford, J.; Tang, J.; Milenković, T. Simultaneous optimization of both node and edge conservation in network alignment via WAVE. International Workshop on Algorithms in Bioinformatics. Springer, 2015, pp. 16-39.

29. Guzzi, P.H.; Milenković, T. Survey of local and global biological network alignment: the need to reconcile the two sides of the same coin. Briefings in Bioinformatics 2017, p. bbw132. 
413 30. Stelzl, U.; Worm, U.; Lalowski, M.; Haenig, C.; Brembeck, F.H.; Goehler, H.; Stroedicke, M.; Zenkner, ${ }_{414}$ M.; Schoenherr, A.; Koeppen, S.; others. A human protein-protein interaction network: a resource for $415 \quad$ annotating the proteome. Cell 2005, 122, 957-968.

416 31. Ibragimov, R.; Malek, M.; Guo, J.; Baumbach, J. GEDEVO: an evolutionary graph edit distance 417 algorithm for biological network alignment. OASIcs-OpenAccess Series in Informatics. Schloss 418 Dagstuhl-Leibniz-Zentrum fuer Informatik, 2013, Vol. 34. 